\title{
Immersion Coefficient for the Marine Optical Buoy (MOBY) Radiance Collectors
}

\author{
Michael Feinholz $^{1}$, B. Carol Johnson ${ }^{2}$, Kenneth Voss ${ }^{3}$, Mark Yarbrough ${ }^{1}$, and Stephanie Flora ${ }^{1}$ \\ ${ }^{1}$ Moss Landing Marine Laboratory, \\ Moss Landing, CA 95039, USA \\ ${ }^{2}$ National Institute of Standards and Technology, \\ Gaithersburg, MD 20899, USA \\ ${ }^{3}$ University of Miami, \\ Coral Gables, FL 33124, USA
}

feinholz@mlml.calstate.edu cjohnson@nist.gov

voss@physics.miami.edu

yarbrough@mlml.calstate.edu

flora@mlml.calstate.edu

The immersion coefficient accounts for the difference in responsivity for a radiometer placed in the air versus water or another medium. In this study, the immersion coefficients for the radiance collectors on the Marine Optical Buoy (MOBY) were modeled and measured. The experiment showed that the immersion coefficient for the MOBY radiance collectors agreed with a simple model using only the index of refraction for water and fused silica. With the results of this experiment, we estimate that the uncertainty in the current value of the immersion coefficient used in the MOBY project is $0.05 \%(k=1)$.

Key words: in-water radiometry; Marine Optical Buoy; ocean color; radiance immersion coefficient; system vicarious calibration.

Accepted: May 9, 2017

Published: June 28, 2017

https://doi.org/10.6028/jres.122.031

\section{Introduction}

The immersion coefficient, $I_{\mathrm{f}}$, is the correction that must be applied to optical radiometers when used in a medium other than the one used for calibration, which is typically air. This factor corrects the calibration coefficient to account for the difference between the instrument operating in air and in water or some other medium. For radiance sensors, the subject of this paper, this immersion coefficient takes into account the differences between the transmission coefficient of the window-medium interface (air or water) and the refractive effects at this interface. The classic solution to this, derived by Austin [1] and reviewed by Mueller and Austin [2], is to use the ratio of the Fresnel coefficients at the interface, along with the square of the index of refraction of the medium, which simplifies to:

$$
I_{\mathrm{f}}=\frac{n_{\mathrm{w}}\left(n_{\mathrm{w}}+n_{\mathrm{g}}\right)^{2}}{\left(1+n_{\mathrm{g}}\right)^{2}},
$$


assuming that the light rays will be hitting the glass window at close to normal incidence, and that the index of refraction of air is 1 . Here, and later in the manuscript, we use the term "glass" (denoted by subscript "g") in a generic sense to represent any window material. In Eq. (1), $n_{\mathrm{w}}$ and $n_{\mathrm{g}}$ are the indices of refraction of water and the glass window, respectively. However, when more complicated optics are used in the system, this value can deviate from this relationship slightly [3] or, as with domed windows, strongly [4]. It is therefore important to experimentally verify that Eq. (1) works for each class or design of radiance sensor [5].

The Marine Optical Buoy (MOBY) [6, 7] is an optical system that has been used for vicarious calibration of virtually every polar-orbiting ocean color sensor since it became operational in 1997 [8-11]. MOBY is moored off the island of Lanai, Hawaii, and has been making measurements of the in-water upwelling radiance, in-water downwelling irradiance, and downwelling surface irradiance almost continuously since this time. Because the fundamental purpose is as a source of vicarious calibration data, there has been a continuous effort to keep the highest possible accuracy in the radiometric data. This includes both a continuous program of improvement of the system and calibration, and understanding and evaluating the measurement uncertainties of the system. While the radiometric uncertainties, and some of the environmentally caused uncertainties, of this system have been described, see Ref. [12], a more recent effort has been to fully document the measurement operation and uncertainties. Using Eq. (1) and the index of refraction for pure water at $30^{\circ} \mathrm{C}[13,14]$ and a fused silica window [15], $I_{\mathrm{f}}=1.72$ at $490 \mathrm{~nm}$, which shows that the $I_{\mathrm{f}}$ corrections are large and must be known with a low uncertainty. As part of the uncertainty effort, an experiment with pure water was performed to validate the immersion coefficient currently used for the MOBY radiance sensors. This paper will describe the MOBY radiance collectors and the results of the experiment to measure the immersion coefficient for this collector.

\section{Experiment Description}

The MOBY system uses a single optical fiber (1 mm core diameter, silica/silica, high $\mathrm{OH}$, and solarization-resistant) to transmit light sequentially from each collector to the measuring spectrometers. For the radiance collector, ambient light goes through a $6.47 \mathrm{~mm}$ thick fused silica window, is reflected from an aluminum mirror at $45^{\circ}$, and then is focused with an $\mathrm{f} / 2$, symmetric convex, $50 \mathrm{~mm}$ focal length synthetic fused silica lens onto the collection fiber. The window and the lens have a $22.5 \mathrm{~mm}$ clear aperture diameter. A schematic of the radiance collector is shown in Fig. 1. The fused silica window is coated with a single layer $\mathrm{MgF}_{2}$ antireflectance coating on the inside surface, the mirror has a protective $\mathrm{SiO}_{2}$ overcoat, and both sides of the lens have a broadband antireflectance coating with maximum reflectance between $400 \mathrm{~nm}$ and $700 \mathrm{~nm}$ of $2.25 \%$. The fiber tip is placed approximately $1.1 \mathrm{~mm}$ in front of the back focal point of the lens, which reduces the effects of inhomogeneities on the front face of the fibers. The full angle field of view of the system in air is $1.73^{\circ}$, reinforcing that the optical rays are normally incident. Recently, with the August 2016 deployment of MOBY261, a wedged quartz depolarizer (DPU-25-A, Thorlabs, Newton, NJ) ${ }^{1}$ was added to the optical train between the mirror and the external window; it is $0.711 \mathrm{~mm}$ behind the window. The $7.35 \mathrm{~mm}$ thick depolarizer has a broadband antireflectance coating from $350 \mathrm{~nm}$ to $700 \mathrm{~nm}$.

\footnotetext{
${ }^{1}$ Certain commercial equipment, instruments, or materials are identified in this paper to foster understanding. Such identification does not imply recommendation or endorsement by the National Institute of Standards and Technology, nor does it imply that the materials or equipment identified are necessarily the best available for the purpose.
} 


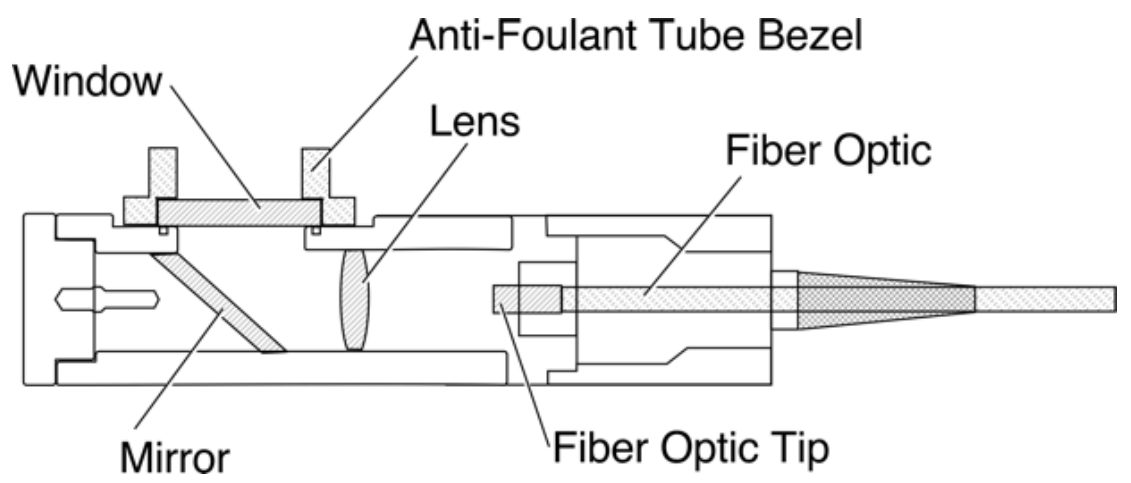

Fig. 1. Schematic of the fiber-coupled radiance collector used at three fixed depths on MOBY.

In the immersion experiment, the collector was arranged facing upwards towards a flat field extended source, approximately $50 \mathrm{~cm}$ by $50 \mathrm{~cm}$ square (Spike-a ${ }^{\mathrm{TM}}$ Flat Fielder, All-Pro Software, Fitchburg, WI), placed $35 \mathrm{~cm}$ from the front surface of the radiance collector. The MOBY collection system as tested was used without the antifouling tube or the depolarizer. There is a lip around the window that is approximately $20 \mathrm{~mm}$ above the window (see Fig. 2). Measurements were done with the space above the radiance collector window dry, and then pure water (high performance liquid chromatography [HPLC] grade Chemical Abstracts Service [CAS] number 7732-18-5, Baxter Burdick \& Jackson, Cat. 365-4) was added to cover the window. Pure water was used in the experiment because the scattering and absorption coefficients are well known, and the tests would be repeatable [16-18]. Various volumes of water, between $2 \mathrm{~mL}$ and $13 \mathrm{~mL}$, were added, and the resulting water depth was calculated. Finally, an additional measurement was done with the window dry. Values of the water depth ranged from $3 \mathrm{~mm}$ to $20 \mathrm{~mm}$. With such small volumes of water, the ultrapurified water was used and discarded after each trial. At each step, measurements were made with the MOBY optical system in a sequence of one dark, five light, and one dark.

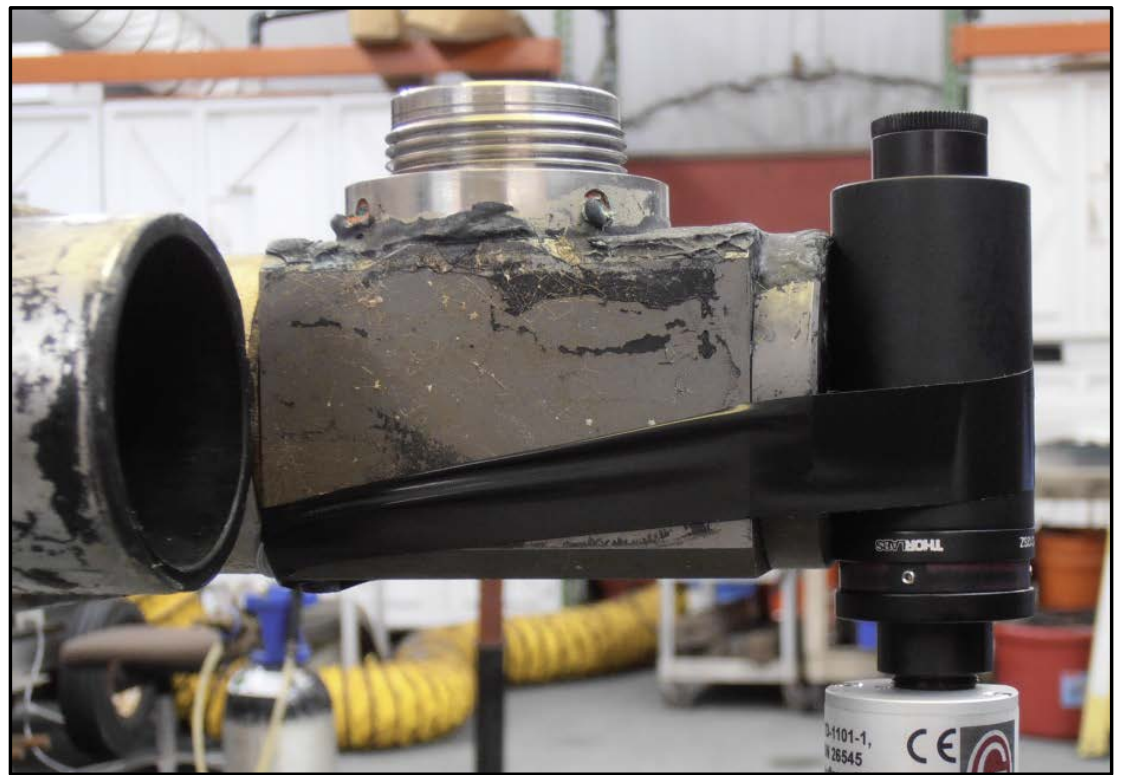

Fig. 2. Photograph of the MOBY radiance collector, which was used without the antifoulant tube, during the setup of the immersion experiment. The shell of the buoy arm is to the left, and the monitor photodiode is to the right. 
An unfiltered, thermoelectric temperature-controlled silicon photodiode (Gigahertz-Optik, Türkenfeld, Germany, TD-1101-1) was used to monitor the Spike-a ${ }^{\mathrm{TM}}$ Flat Fielder during the MOBY experiments (seen to the right in Fig. 2). The panel was allowed to warm up for $1 \mathrm{~h}$ before MOBY measurements began. Three repeats were done, each taking about $1 \mathrm{~h}$ to perform and consisting of 4 to 12 different depths, and lasting between $17 \mathrm{~min}$ and $34 \mathrm{~min}$. The net signals were corrected for temperature sensitivity in the MOBY spectrographs, termed the Marine Optical System (MOS), and the spectrally integrated drift in the Spike-a ${ }^{\mathrm{TM}}$ Flat Fielder source as determined by the monitor photodiode. These corrected dry-before and dry-after measurements indicate a spectral dependence that we attribute to the Spike-a ${ }^{\mathrm{TM}}$ Flat Fielder source, with maximum values of $0.98 \%$ at $450 \mathrm{~nm}, 0.83 \%$ at $500 \mathrm{~nm}, 0.41 \%$ at $600 \mathrm{~nm}$, and negligible change at $700 \mathrm{~nm}$. These differences were treated as linear drift at each wavelength during the set of depths and used to correct the results to the time of measurement for each water depth.

The experimental uncertainty consists of the measurement uncertainty and the uncertainty due to the temperature and drift corrections. For each water depth, the MOS signal-to-noise ratios were greater than 100:1 between $425 \mathrm{~nm}$ and $725 \mathrm{~nm}$, and these values were used to determine the measurement uncertainty. The MOS temperature changed by about $0.15^{\circ} \mathrm{C}$ in $33 \mathrm{~min}$, the thermal correction required was less than $0.08 \%$ in the net signal, and we assumed an uncertainty of $0.08 \%$ for this factor. The photodiode measured drift was between $-0.01 \%$ and $0.06 \%$ over each series, with an uncertainty in this correction between $0.008 \%$ and $0.013 \%$. The uncertainty in the dry-before and dry-after correction was determined using the maximum observed change and a uniform distribution. The combined experimental standard uncertainty for each measured signal ratio $S_{\text {wet }} / S_{\text {dry }}$ [see Eq. (10) below], is $0.52 \%$ at $450 \mathrm{~nm}, 0.59 \%$ at $500 \mathrm{~nm}$, $0.30 \%$ at $600 \mathrm{~nm}$, and $0.58 \%$ at $700 \mathrm{~nm}$.

Because the light source stayed in the air, above the surface of the water, and because the layer of water was very thin, this experiment did not directly result in the immersion coefficient. We can model the interreflections such that if our model agrees with the experiment, we can expect our model of the immersion coefficient to be correct for the MOBY radiance collector. Figure 3 illustrates the light paths in the experiment. For simplicity, we have separated the light paths of the air/water/glass/air system into three parts, illustrating the transmittance of the water layer, the reflection of light back into the glass due to the water layer, and the transmittance of the glass layer. In Fig. 3 and the equations that follow, $T_{x}$ is the internal transmission through the $x$ layer, $t_{x y}$ and $r_{x y}$ are the transmission and reflection at the $x-y$ interface, and $T_{x y z}$ is the transmission through the $y$ medium when surrounded by medium $x$ and medium $z$.

The water and glass layers, because they are thin, can have interreflections inside these layers. Following Ref. [19] in the water layer, the amount of light transmitted through the water layer, for incoherent light, is given by

$$
T_{\mathrm{awg}}=t_{\mathrm{aw}} T_{\mathrm{w}} t_{\mathrm{wg}}\left[1+r_{\mathrm{wg}} T_{\mathrm{w}}^{2} r_{\mathrm{wa}}+\left(r_{\mathrm{wg}} T_{\mathrm{w}}^{2} r_{\mathrm{wa}}\right)^{2}+\ldots\right] .
$$

The terms in the series are much smaller than unity, and the series converges quickly, so $T_{\text {awg }}$ can be expressed exactly as [20]

$$
T_{\mathrm{awg}}=\frac{t_{\mathrm{aw}} T_{\mathrm{w}} t_{\mathrm{wg}}}{1-r_{\mathrm{wg}} T_{\mathrm{w}}^{2} r_{\mathrm{wa}}}
$$

These factors depend on wavelength, which has not been shown explicitly. Application of the Fresnel equations at normal incidence gives the transmittance and reflectance as a function of index of refraction: $r_{x y}=\left(\left(n_{x}-n_{y}\right) /\left(n_{x}+n_{y}\right)\right)^{2}$ and $t_{x y}=1-r_{x y}$ [21]. If light is reflected upwards into this water layer from the glass layer below, the reflectance back to the glass layer is:

$$
R_{\mathrm{gwa}}=t_{\mathrm{gw}} T_{\mathrm{w}} r_{\mathrm{wa}} T_{\mathrm{w}} t_{\mathrm{gw}}\left[1+r_{\mathrm{wg}} T_{\mathrm{w}}^{2} r_{\mathrm{wa}}+\left(r_{\mathrm{wg}} T_{\mathrm{w}}^{2} r_{\mathrm{wa}}\right)^{2}+\ldots\right]
$$



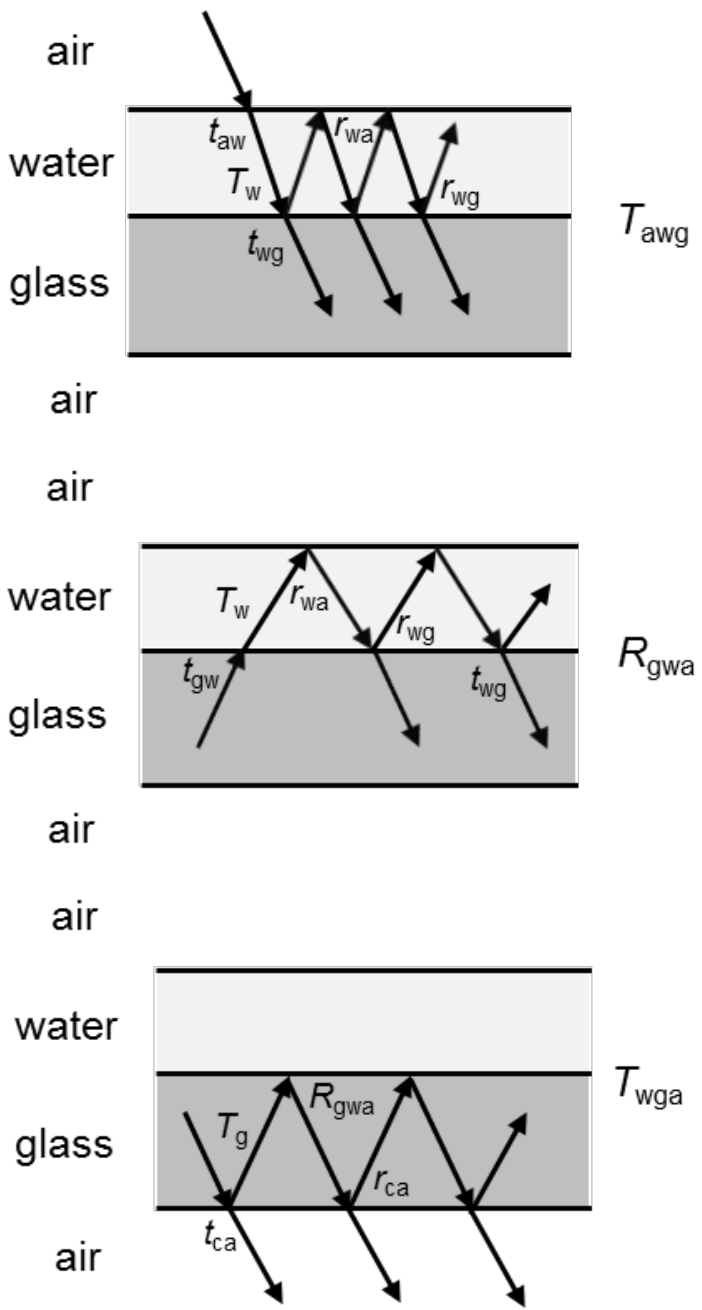

Fig. 3. Illustration of light paths in the experiment, showing the different interreflections that can occur in each layer. This diagram is not to scale, and the mirror, lens, and fiber are located $24.1 \mathrm{~mm}, 50.4 \mathrm{~mm}$, and $97.4 \mathrm{~mm}$, respectively, behind the window; see also Fig. 1. Thus, no interreflections are expected between components and the glass window.

$$
R_{\mathrm{gwa}}=\frac{t_{\mathrm{gw}}^{2} T_{\mathrm{w}}^{2} r_{\mathrm{wa}}}{1-r_{\mathrm{wg}} T_{\mathrm{w}}^{2} r_{\mathrm{wa}}} .
$$

Similar to the water layer, the glass layer will transmit light with interreflections inside this layer. However, to allow the case of light being transmitted back into the water layer, and then reflecting back down, instead of using $r_{\mathrm{gw}}$ for the reflection at the glass-water interface, we use $R_{\mathrm{gwa}}$ derived above:

$$
T_{\text {wga }}=T_{\mathrm{g}} t_{\mathrm{ca}}\left[1+r_{\mathrm{ca}} T_{\mathrm{g}}^{2} R_{\mathrm{gwa}}+\left(r_{\mathrm{ca}} T_{\mathrm{g}}^{2} R_{\mathrm{gwa}}\right)^{2}+\ldots\right]
$$

or

$$
T_{\text {wga }}=\frac{T_{\mathrm{g}} t_{\mathrm{ca}}}{1-r_{\mathrm{ca}} T_{\mathrm{g}}^{2} R_{\mathrm{gwa}}} .
$$


In these last two equations, $r_{\mathrm{ca}}$ and $t_{\mathrm{ca}}$ represent the reflectance and transmittance, respectively, of the coated glass-air interface, rather than a simple glass-air interface. Interreflections may be possible between the back of the window and the mirror-lens-fiber system, but since the lens is coated with an antireflectance layer, is significantly behind the glass, and is curved (and given the angular acceptance of the system), it is unlikely that these reflections will be significant, and thus they are ignored.

Following the same approach as above, the transmittance of the window when dry is

$$
T_{\text {aga }}=\frac{t_{\mathrm{ag}} T_{\mathrm{g}} t_{\mathrm{ca}}}{\left(1-r_{\mathrm{ga}} T_{\mathrm{g}}^{2} r_{\mathrm{ca}}\right)}
$$

The schematic for the light paths of the dry window is illustrated in Fig. 4.

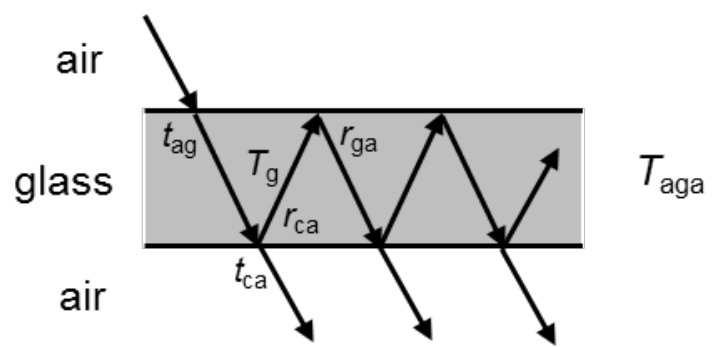

Fig. 4. Illustration of light paths for the dry window situation, showing the different interreflections that can occur. This diagram is not to scale, and, as in Fig. 3, the ray angles are schematically drawn and do not reflect the physical situation.

If the model is correct, in this experiment the ratio of the signal when the window is immersed to when the window is dry will be:

$$
S_{\text {Wet }} / S_{\text {Dry }}=\frac{T_{\text {awg }} T_{\text {wga }}}{T_{\text {aga }}}=\frac{t_{\mathrm{aw}} T_{\mathrm{w}} t_{\mathrm{wg}}}{\left(1-r_{\mathrm{wg}} T_{\mathrm{w}}^{2} r_{\mathrm{wa}}\right)} \frac{T_{\mathrm{g}} t_{\mathrm{ca}}}{\left(1-r_{\mathrm{ca}} T_{\mathrm{g}}^{2} R_{\mathrm{gwa}}\right)} \frac{\left(1-r_{\mathrm{ga}} T_{\mathrm{g}}^{2} r_{\mathrm{ca}}\right)}{t_{\mathrm{ag}} T_{\mathrm{g}} t_{\mathrm{ca}}} .
$$

For the wavelengths we are considering, and with a $6.47 \mathrm{~mm}$ thick fused silica window, $T_{g}=1$ [22]. So, simplifying the above equation:

$$
S_{\text {Wet }} / S_{\text {Dry }}=\frac{T_{\text {awg }} T_{\text {wga }}}{T_{\text {aga }}}=\frac{t_{\mathrm{aw}} T_{\mathrm{w}} t_{\mathrm{wg}}\left(1-r_{\mathrm{ga}} r_{\mathrm{ca}}\right)}{t_{\mathrm{ag}}\left(1-r_{\mathrm{wg}} T_{\mathrm{w}}^{2} r_{\mathrm{wa}}\right)\left(1-r_{\mathrm{ca}} R_{\mathrm{gwa}}\right)}
$$

The transmission of the pure water can be calculated by combining the scattering coefficient [17] and absorption coefficient of pure water [16, 18]. With the index of refraction for the fused silica window [15], and for pure water $[13,14]$, we can calculate the expected ratio.

\section{Results}

Figure 5 shows a comparison of the experimental measurements and the modeled experiment, using Eq. (10). The roll-off at longer wavelengths is due to water absorption. The increased noise at different spectral regions is directly related to the spectral output of the flat field extended source, which uses lightemitting diodes for illumination. At very small water thicknesses ( $3 \mathrm{~mm}$ to $6 \mathrm{~mm}$ ), there is a small offset ( $0.3 \%$ to $0.5 \%$ ) between the experiment and model results. This is within the estimated combined standard uncertainty of the experimental data and its model, Eq. (10). At depths of $8 \mathrm{~mm}$ or greater, the model and data agree almost exactly, with an average difference of $0.00024 \pm 0.0013$. For the shallow depths, there may be problems due to the surface tension of the water and possible curvature of the air-water surface meniscus, which would reduce the interreflection at that interface. However, neglecting the terms due to the interreflection of light in the water layer does not change the result significantly. 

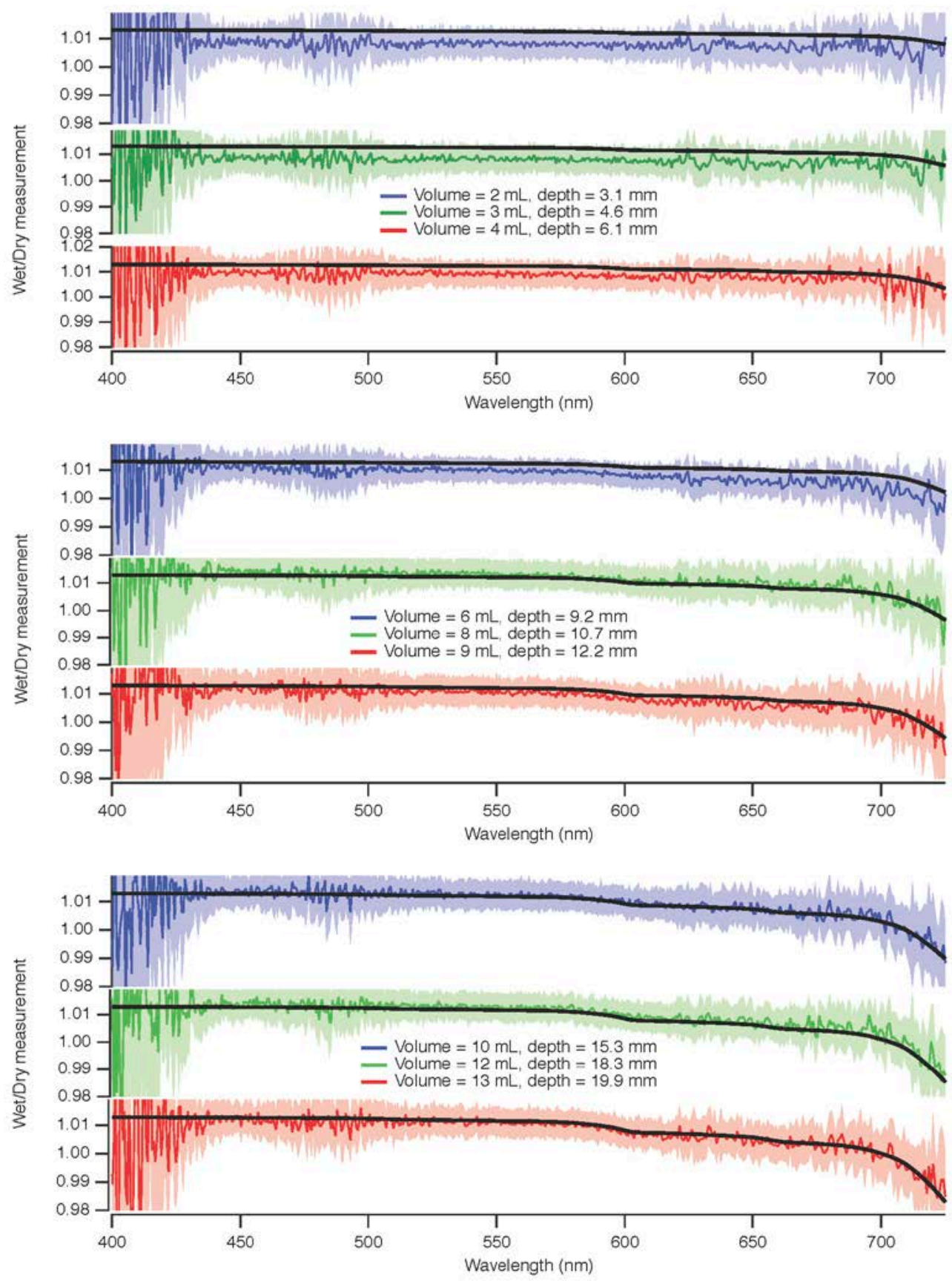

Fig. 5. Comparison of model result (black line) and experimental data (thin lines, where shaded area indicates $k=1$ uncertainty). For the thinnest layers, the data are slightly smaller but within the uncertainty estimates. For layers $9 \mathrm{~mm}$ and thicker, the agreement is very good.

\section{Application to the MOBY Radiance Collectors}

As shown by Ref. [2], the immersion coefficient is the product of the field-of-view ratio $n_{\mathrm{w}}^{2} / n_{a}^{2}$ and the ratio of the in-air to in-water transmittance. The field-of-view ratio follows from the $n^{2}$ law of radiance, 
which is exact if there are no losses at the medium to window surface, e.g., scattering from imperfections. If we include in the transmittance term the interreflections of the light in the window, and we also include the index of refraction of air $\left(n_{\mathrm{a}}\right)$, Eq. (1) (using the expansions similar to above) becomes:

$$
I_{\mathrm{f}}=\frac{n_{\mathrm{w}}^{2}}{n_{\mathrm{a}}^{2}} \frac{t_{\mathrm{ag}}\left(1-r_{\mathrm{ca}} r_{\mathrm{wg}}\right)}{t_{\mathrm{wg}}\left(1-r_{\mathrm{ca}} r_{\mathrm{ag}}\right)} .
$$

The difference between Eq. (1) and Eq. (11) is $0.012 \%$ [where Eq. (11) is higher] at $500 \mathrm{~nm}$ and at a salinity of $32 \mathrm{~g} / \mathrm{kg}$ and a seawater temperature of $20^{\circ} \mathrm{C}$. Equation (11) varies by $-0.017 \%$ per degree Celsius between $20^{\circ} \mathrm{C}$ and $30^{\circ} \mathrm{C}$ and $0.027 \%$ per salinity mass fraction in gram per kilograms between 30 $\mathrm{g} / \mathrm{kg}$ and $40 \mathrm{~g} / \mathrm{kg}$ salinity. The temperature at the MOBY site varies from $23^{\circ} \mathrm{C}$ to $30{ }^{\circ} \mathrm{C}$, and the salinity range is $33 \mathrm{~g} / \mathrm{kg}$ to $36 \mathrm{~g} / \mathrm{kg}$; thus, $I_{\mathrm{f}}$ can be considered a constant with regard to temperature and salinity but variable with regard to wavelength at this site.

The immersion coefficient was also investigated using Zemax (Zemax, LLC, Kirkland, WA), a commercial optical design software package, to model the MOBY radiance collector for the in-air and inwater cases. The field of views were determined from the vignetting curves and the transmittance was determined from the inputs of the optical model. The agreement with Eq. (1) at $550 \mathrm{~nm}$ was $0.07 \%$. We also modeled the optical system using a matrix representation to investigate the effect on $I_{\mathrm{f}}$ from the positioning of the fiber tip $1.1 \mathrm{~mm}$ from the lens focal point, and saw that there was no effect.

\section{Conclusions}

This agreement of the experiment results and our model of the experiment shows that we can model the immersion coefficient correctly, and the coefficient depends only on the index of refraction of the water, the window coating, and fused silica.

This experiment validates the use of the theoretical values for $I_{\mathrm{f}}$ in the MOBY data set, as the results clearly agree within the uncertainties. The simple optical design of the MOBY radiance collector facilitated accurate modeling of $I_{\mathrm{f}}$, a result that could be applied to the design of in-water, ocean color radiometers.

Currently, the MOBY project uses Eq. (1) in deriving $I_{\mathrm{f}}$. Equation (11) is $0.012 \%$ larger than Eq. (1) when all of the interreflections in the quartz window are included. Because the quartz is coated, there are few interreflections in the quartz, and so Eq. (11) and Eq. (1) give similar results. The main difference is in using the true air refractive index in Eq. (11). We will continue to use Eq. (1) in the MOBY project, and we estimate the standard uncertainty in this factor, applied with an average salinity and temperature, to be $0.05 \%$, mostly driven by the range of salinities and temperatures historically recorded at the location of MOBY. In the future, we will assess the impact of the depolarizer that was added in 2016 on $I_{\mathrm{f}}$ and its uncertainty.

\section{Acknowledgments}

We want to acknowledge Dennis Clark's guidance and ideas in this work. This technique for measuring the immersion coefficient was his idea but was not implemented, unfortunately, until after he had passed away. We gratefully acknowledge Clarence Zarobila of NIST for the optical modeling of the $L_{u}$ collector using Zemax. This work was supported by the National Oceanic and Atmospheric Administration (NOAA) under grant NA15OAR4320064 and by the National Aeronautics and Space Administration (NASA) under grant NNX14AP63G.

\section{References}

[1] Austin RW (1976) Air-water radiance calibration factor. in Technical Memorandum ML-76-004t (Scripps Institution of Oceanography, La Jolla, CA), pp 1 - 8.

[2] Mueller JL, Austin RW (2003) Characterization of Oceangraphic and Atmospheric Radiometers. Ocean Optics Protocols for Satellite Ocean Color Sensor Validation, Revision 4, Volume II: Instrument Specifications, Characterization, and 
Center, National Aeronautics and Sapce Administration, Greenbelt, Maryland), pp 17 - 33.

[3] Ohde T, Siegel H (2003) Derivation of immersion factors for the hyperspectral TriOS radiance sensor. Journal of Optics A: Pure and Applied Optics 5:L12 - L14. https://doi.org/10.1088/1464-4258/5/3/103

[4] Voss KJ, Chapin AL (2005) Upwelling radiance distribution camera system, NURADS. Optics Express 13:4250 - 4262. https://doi.org/10.1364/OPEX.13.004250

[5] Zibordi G, Darecki M (2006) Immersion factors for the RAMSES series of hyper-spectral underwater radiometers. Journal of Optics A: Pure and Applied Optics 8:252 - 258. https://doi.org/10.1088/1464-4258/8/3/005

[6] Clark DK, Gordon HR, Voss KJ, Ge Y, Broenkow W, Trees C (1997) Validation of atmospheric corrections over the oceans. J Geophys Res 102:17209 - 17217. https://doi.org/10.1029/96JD03345

[7] Clark DK, Yarbrough MA, Feinholz ME, Flora SJ, Broenkow W, Kim YS, Johnson BC, Brown SW, Yuen M, Mueller JL (2002) MOBY, a radiometric buoy for performance monitoring and vicarious calibration of satellite ocean color sensors: measurement and data analysis protocols. Ocean Optics Protocols for Satellite Ocean Color Sensor Validation, Revision 3, Volume 2, Vol. NASA/TM--2002-21004, eds Mueller JL \& Fargion GS (NASA Goddard Space Flight Center, Greenbelt, MD), pp 138 - 170.

[8] Barnes RA, Eplee RE, Schmidt GM, Patt FS, McClain CR (2001) Calibration of SeaWiFS. I. Direct techniques. Appl Optics 40:6682 - 6700. https://doi.org/10.1364/AO.40.006682

[9] Eplee RE, Robinson WD, Bailey SW, Clark DK, Werdell PJ, Wang M, Barnes RA, McClain CR (2001) Calibration of SeaWiFS. II. Vicarious techniques. Appl Optics 40:6701 - 6718. https://doi.org/10.1364/AO.40.006701

[10] Franz BA, Bailey SW, Werdell PJ, McClain CR (2007) Sensor-independent approach to the vicarious calibration of satellite ocean color radiometry. Appl Opt 46:5068 - 5082. https://doi.org/10.1364/AO.46.005068

[11] Wang M, Liu X, Tan L, Jiang L, Son SH, Shi W, Rausch K, Voss K (2013) Impacts of VIIRS SDR performance on ocean color products. J Geophys Res Atmos 118:10347 - 10360. https://doi.org/10.1002/jgrd.50793

[12] Brown SW, Flora SJ, Feinholz ME, Yarbrough MA, Houlihan T, Peters D, Kim YS, Mueller JL, Johnson BC, Clark DK (2007) The Marine Optical Buoy (MOBY) radiometric calibration and uncertainty budget for ocean color satellite sensor vicarious calibration. Proc SPIE 6744:67441M. https://doi.org/10.1117/12.737400

[13] Austin RW, Halikas G (1976) The Index of Refraction of Seawater, Vol. SIO Ref. No. 76-1, p 64.

[14] Quan X, Fry ES (1995) Empirical equation for the index of refraction of seawater. Appl Optics 34:3477 - 3480. https://doi.org/10.1364/AO.34.003477

[15] Kitamura R, Pilon L, Jonasz M (2007) Optical constants of silica glass from extreme ultraviolet to far infrared at near room temperature. Appl Optics 46:8118 - 8133. https://doi.org/10.1364/AO.46.008118

[16] Lee Z, Wei J, Voss K, Lewis M, Bricaud A, Huot Y (2015) Hyperspectral absorption coefficient of “pure” seawater in the range of 350-550 nm inverted from remote sensing reflectance. Appl Optics 54:546 - 558. https://doi.org/10.1364/AO.54.000546

[17] Morel A (1974) Optical properties of pure water and pure seawater. Optical Aspects of Oceanography, eds Jerlov NG \& Nielson ES (Academic Press, New York), pp 1 - 24.

[18] Pope RM, Fry ES (1997) Absorption spectrum (380-700 nm) of pure water. II. Integrating cavity measurements. Appl Optics 36:8710 - 8723. https://doi.org/10.1364/AO.36.008710

[19] Zibordi G (2006) Immersion factor of in-water radiance sensors: assessment for a class of radiometers. $J$ Atmospheric and Oceanic Technology 23:302 - 313. https://doi.org/10.1175/JTECH1847.1

[20] Abramowitz M, Stegun IA (1964) Handbook of Mathematical Functions: with Formulas, Graphs, and Mathematical Tables (US Department of Commerce, Washington, D.C.), Vol. p 1046.

[21] Jackson JD (1999) Classical Electrodynamics, Third Edition (John Wiley \& Sons, New York, NY), Vol. p 815.

[22] Edwards OJ (1966) Optical absorption coefficient of fused silica in wavelength range 0.17 to 3.5 microns from room temperature to $980 \operatorname{deg}$ C (Washington, D.C.), Vol. NASA-TN-D-3257, p 25.

About the authors: Michael Feinholz is the calibration scientist for MOBY. B. Carol Johnson is a physicist in the Sensor Science Division at NIST. Kenneth Voss is a professor in the Physics Department at the University of Miami. Mark Yarbrough is the principle investigator of the MOBY Project. Stephanie Flora is an analyst and programmer for the MOBY Project.

The National Institute of Standards and Technology is an agency of the U.S. Department of Commerce. 